\title{
Robotically Assisted Interventions: Clinical Trial for Spinal Blocks
}

\author{
Kevin Cleary ${ }^{1}$, Vance Watson ${ }^{1}$, David Lindisch ${ }^{1}$, Alexandru Patriciu ${ }^{2}$, \\ Dumitru Mazilu², and Dan Stoianovici² \\ ${ }^{1}$ Imaging Science and Information Systems (ISIS) Center, Department of Radiology, \\ Georgetown University Hospital, Washington, DC, USA \\ \{cleary@georgetown.edu\} \\ ${ }^{2}$ Urobotics Laboratory, Johns Hopkins Medical Institutions, Baltimore, MD, USA
}

\begin{abstract}
Percutaneous interventions are performed by freehand passages of instruments, such as needles, from the skin surface to the anatomy of interest. The main problem with this approach is that the physician can be inaccurate in aligning the instrument and staying on course. A joystick-controlled robotic needle driver may allow the physician to more precisely target the anatomy. This paper describes our experience with a robotic needle driver in a 20-patient clinical trial of nerve and facet blocks. Our next stage of research in robotically assisted lung biopsy is also mentioned.
\end{abstract}

\section{Materials and Methods}

The robotic needle driver consists of a three degree-of-freedom (DOF) translational stage, a 2-DOF rotational stage which can orient the needle to any angle, and a 1DOF needle drive mechanism [1]. The robot is controlled using a joystick and touch screen. The interventionalist can thus manipulate the needle under $\mathrm{x}$-ray fluoroscopy without direct exposure to the radiation beam. After cadaver studies using the robot to precisely position a needle in the lumbar spine were successfully completed [2], a randomized clinical trial of 20 patients undergoing nerve and facet blocks was approved by the FDA and the local institutional review board. The procedure is done in the standard manner except the robot is used to position, orient, and drive the needle under physician control. A/P fluoroscopy is used to position and orient the needle, and lateral fluoroscopy is used to monitor the depth of insertion.

The robot is mounted on the interventional table using a custom-designed locking mechanism. The robot is positioned initially near the skin entry point by loosening the passive gross positioning mechanism and moving the needle driver end of the robot by hand. Once this initial position has been attained, the mechanism is locked and the robot is switched to operate by physician control.

\section{Results}

The study was approved by the local Institutional Review Board and the Food and Drug Administration (FDA) and was conducted from August to December, 2002. The study was completed by a single fellowship trained interventional neuroradiologist at Georgetown University Hospital using a Siemens Neurostar bi-plane fluoroscopy system. The standard manual technique was used on 10 patients and the robotic de 
vice was used on 10 patients. The patients ranged in age from 30 to 70 years. The spine levels were from S-1 to L-5. No complications were observed in the study. One of the patients in the robotics arm had to be converted to a manual procedure due to slippage of the needle driver. This conversion was done without difficulty or complications.

There were two outcome measures: 1) accuracy of needle placement and 2) pain relief. Accuracy of needle placement was determined as follows. Before the interventionalist began placing the needle, both an $\mathrm{A} / \mathrm{P}$ and lateral image of the patient were obtained. The interventionalist would then annotate each image with an arrow to indicate the desired target location of the needle (the interventionalist was not blinded as to manual/robotic technique as this was not practical). After the needle was placed, an $\mathrm{A} / \mathrm{P}$ and lateral image was again obtained. The two sets of images were compared to determine the distance between the intended location of the needle and the actual location of the needle. Pain relief was measured using a visual-analog scale, with 0 representing no pain and 10 representing excruciating pain.

\section{Discussion}

The results to date show that is feasible to use a joystick controlled robot for nerve and facet blocks. While this was a pilot study and not enough data was gathered for statistical significance, some general trends can be observed. The mean accuracy in the robot $(1.105 \mathrm{~mm})$ and manual $(1.238 \mathrm{~mm})$ is about the same. Therefore, it appears that the robot is capable of accurate needle placement.

As expected, the pain score post-treatment was significantly less than the pain score pre-treatment in both the robot and manual arms. In the robot arm, pain scores fell from a mean of 6.3 pre-treatment to 1.8 post-treatment. In the manual arm, pain scores fell from 6.0 pre-treatment to 0.9 post-treatment. Patients had to sign an informed consent form and were generally receptive to the use of the robot.

\section{Future Work}

We are in the process of submitting our progress report to the FDA and asking for permission to enroll additional patients. In addition, the next clinical application that we are investigating is lung biopsy. The goal is to use the robotic system to assist the physician in accurate computed tomography (CT) fluoroscopy-guided needle biopsy of lung nodules.

Acknowledgements. This work was funded by U.S. Army grant DAMD17-99-19022. The content of this manuscript does not reflect the position or policy of the U.S. Government.

\section{References}

1. D. Stoianovici, K. Cleary, A. Patriciu, D. Mazilu, A. Stanimir, N. Craciunoiu, V. Watson, L. Kavoussi, “AcuBot: A Robot for Radiological Interventions," accepted for publication in IEEE Transactions on Robotics and Automation, May 2003.

2. K. Cleary., D. Stoianovici, A. Patriciu, D. Mazilu, D. Lindisch and V. Watson (2002). "Robotically assisted nerve and facet blocks: a cadaveric study." Acad Radiol 9(7): 821-5. 